\title{
Lidil
}

Revue de linguistique et de didactique des langues

$63 \mid 2021$

Littératie numérique et didactique des langues et des cultures

\section{Françoise Boch, Catherine Frier et Fanny Rinck (dir.), Littéracie et démarches pédagogiques \\ engageantes, revue Le français aujourd'hui, $\mathrm{n}^{\circ} 212$ 2021, 144 p.}

\section{Valeria Villa-Perez}

\section{OpenEdition}

\section{Journals}

Édition électronique

URL : http://journals.openedition.org/lidil/9096

DOI : 10.4000/lidil.9096

ISSN : 1960-6052

\section{Éditeur}

UGA Éditions/Université Grenoble Alpes

\section{Édition imprimée}

ISBN : 978-2-37747-283-3

ISSN : 1146-6480

Référence électronique

Valeria Villa-Perez, « Françoise Boch, Catherine Frier et Fanny Rinck (dir.), Littéracie et démarches pédagogiques engageantes, revue Le français aujourd'hui, n 212 », Lidil [En ligne], 63 | 2021, mis en ligne le 30 avril 2021, consulté le 30 avril 2021. URL : http://journals.openedition.org/lidil/9096 ; DOI https://doi.org/10.4000/lidil.9096

Ce document a été généré automatiquement le 30 avril 2021.

(C) Lidil 


\section{Françoise Boch, Catherine Frier et Fanny Rinck (dir.), Littéracie et démarches pédagogiques engageantes, revue Le français aujourd'hui, $\mathrm{n}^{\circ} 212$}

2021,144 p.

Valeria Villa-Perez

\section{RÉFÉRENCE}

Françoise Boch, Catherine Frier et Fanny Rinck (dir.), Littéracie et démarches pédagogiques engageantes, revue Le français aujourd'hui, $\mathrm{n}^{\circ}$ 212, 2021, $144 \mathrm{p}$.

1 S'inscrivant dans une approche « ethnolittéracique » (Rispail, 2020), le dernier numéro du Français aujourd'hui, dirigé par F. Boch, C. Frier et F. Rinck, rappelle les enjeux sociaux forts de la littéracie et sa fonction émancipatrice en pédagogie.

2 Les auteures soulèvent en introduction des questions vives telles que l'accès inégal à l'écrit, les difficultés d'apprentissage de la lecture présentes encore en fin de scolarité obligatoire, la montée en puissance du recours à l'écrit dans la vie quotidienne et professionnelle (cf. Lahire, 1993, La raison des plus faibles. Sur le rapport au travail, écritures domestiques et lectures en milieux populaire), et l'augmentation constante de «la part langagière du travail», comme le montre un article de J. Boutet(2001). Elles s'interrogent sur les enjeux liés à l'inclusion scolaire de publics variés plurilingues car issus de parcours migratoires ou des élèves pour lesquels la transmission du capital littéracique ne se réalise pas au niveau familial et « qui n'ont pas reçu les codes d'accès de la culture écrite [...] et les comportements sociaux, valorisés scolairement, qui s'y rapportent» (p. 6).

3 Ces constats liminaires laissent ensuite la place à un bouquet de contributions qui conjuguent des pratiques pédagogiques, encourageant l'émergence de "postures 
littéraciques» (cf. Rispail, 2020, citée par les coordinatrices), avec la notion d'engagement de l'enseignant.e, du.de la formateur.trice, de la famille et de l'élève dans une optique de co-construction des savoirs. Organisée en trois parties, la publication réunit neuf articles et nous avons choisi de mettre l'accent sur certains d'entre eux, pour la variété des contextes géographiques, formels ou informels qu'ils présentent.

4 P. Sirois, A. Vanlint et A.-S. Gravel illustrent les résultats d'une étude longitudinale réalisée dans une école primaire, en classe ordinaire à Montréal, à partir d'activités d'écriture créative. Les enseignants s'appuient sur les compétences déjà-là et en devenir ainsi que sur la prise en compte des représentations des enfants sur l'écriture. $\mathrm{Si}$ on problématise souvent les difficultés des élèves, on montre moins le besoin d'accompagnement des enseignant.e.s dont l'insécurité face à l'apprentissage de l'écriture est fréquente.

5 J.-F. Boudet, É. Clayette et P. Salam présentent un projet d'ateliers créatifs (2013-2018) réalisé à l'Université du Mans. Fondés sur une participation volontaire et/ou obligatoire, selon le stade de la formation, ces ateliers à destination des étudiant.e.s de licence de langues, STAPS (Sciences et techniques des activités physiques et sportives) et droit, visent à renforcer leurs compétences à l'écrit. L'appropriation de genres textuels académiques nouveaux s'avère complexe et la réflexion sur une démarche en trois phases : un temps d'étrangeté, d'apprentissage puis d'affiliation (p. 94) est féconde pour consolider des compétences littéraciques trop souvent considérées comme acquises à ce stade du parcours formatif.

6 M. Wibrin décrit son observation participante d'une expérience de soutien à la lecture menée en Belgique, par un groupe de mères réunies au sein de l'association de l'école primaire de leurs enfants. Conçu pour épauler des enfants de milieux populaires scolarisés dans un réseau d'éducation prioritaire, le projet s'est transformé en un instrument d'empowerment pour des parents à capital culturel peu adapté à l'école, investis dans une démarche collaborative. La chercheure analyse le rôle joué par des pratiques littéraciques qui, en évitant les conflits de loyauté entre les enfants et leur famille, «n'émancipent pas l'enfant de son milieu familial, mais [...] émancipent l'enfant et son milieu familial » (p. 106).

7 Enfin l'article de A. Fabulet, animatrice de l'atelier, et de C. Vorger, chercheure, rend compte du travail original accompli en France au sein d'ateliers philo en maternelle, slam pour des élèves dits "allophones». L'approche inclusive privilégiée unit des modalités d'expression différentes et invite les apprenants à s'engager également à travers le corps, ce qui assouplit le carcan de l'écrit. Cette approche prend ses distances avec une conduite prescriptive et normative: "L'école enseigne la syntaxe, l'orthographe, la grammaire. Elle modèle la forme. [...] L'animatrice [...] modifie d'emblée ces règles. L'utilisation commune du langage va être ici bousculée. Les « formes » intégrées seront un appui, plus tard, lorsqu'il s'agira de consolider le texte. » (p. 121)

8 Le numéro coordonné par F. Boch, C. Frier et F. Rinck - entre autres par la richesse des contextes francophones géographiques (France, Québec, Belgique...) et la diversité des acteurs et niveaux de scolarité décrits - est une contribution importante aux études sur les littéracies. D'aucuns y verront une publication qui met en évidence les modalités d'appropriation littéracique à l'école en tant que projet collectif et sociétal où chacun.e participe à sa manière au renforcement du pouvoir d'agir. Le fait de donner la parole aux acteurs de terrain, dont le travail reste souvent dans l'ombre, en est une 
illustration. D'autres y verront une manière d'articuler littéracies et langues d'origine et/ou d'héritage des élèves, notamment en contexte plurilingue (même si les contributions portant sur ce binôme sont moins nombreuses, cf. par exemple le travail de C. Maynard), ce qui peut être considéré comme une forme d'engagement des enseignant.e.s et potentiellement un outil scolaire pour réduire les faiblesses littéraciques.

9 Au vu des enjeux sociétaux inévitablement liés aux littéracies, pour la réduction des inégalités par exemple, cette publication qui peut intéresser enseignant.e.s et chercheur.e.s contribue solidement à l'élargissement des questionnements dans ce domaine.

\section{AUTEURS}

\section{VALERIA VILLA-PEREZ}

ECLLA, Université Jean Monnet Saint-Étienne 